\title{
Overexpression of the Small Heat Shock Protein, PtsHSP19.3 from Marine Red Algae, Pyropia tenera (Bangiales, Rhodophyta) Enhances Abiotic Stress Tolerance in Chlamydomonas
}

\author{
Yujin Jin $\cdot$ Sungwhan Yang $\cdot$ Sungoh Im $\cdot$ Won-Joong Jeong $\cdot$ EunJeong Park $\cdot$ Dong-Woog Choi
}

Received: 21 June 2017 / Revised: 3 August 2017 / Accepted: 22 August 2017

(c) Korean Society for Plant Biotechnology

\begin{abstract}
Water temperature is one of the major factors that impacts the growth and life cycle of Pyropia tenera, one of the most valuable and cultivated marine red algae belonging to Bangiales (Rhodophytes). We analyzed transcriptome from gametophyte of $P$. tenera under normal and high temperature conditions, and identified four small heat shock proteins (sHSPs). They have no significant amino acid sequence homology with known proteins in public databases except PhsHSP22 from Pyropia haitanensis. PtsHSP19.3 gene responded to high temperature but slightly or not to desiccation, freezing or high salt condition. When the PtsHSP19.3 gene was overexpressed in Chlamydomonas reinhardtii, transformed Chlamydomonas lines revealed much higher growth rate than that of control cells under heat stress condition. Transformed cells also grew well in those of the control cell onto the medium containing high salt or $\mathrm{H}_{2} \mathrm{O}_{2}$. When the PtsHSP19.3 was fused to GFP and introduced into tobacco protoplast, fluorescence was detected at several spots. Results indicate that PtsHSP19.3 may form super-molecular assembles and be involved in tolerance to heat stress.
\end{abstract}

Keywords Red algae, Pyropia tenera, small heat shock protein, Heat tolerance, Abiotic stress tolerance

Y. J. Jin $\cdot$ S. W. Yang $\cdot$ S. O. Im $\cdot$ D.-W. Choi $(ه)$ Department of Biology Education and Kumho Life Science Laboratory, Chonnam National University, Gwangju, 61186, Korea e-mail: dwchoi63@jnu.ac.kr

W.-J. Jeong

Plant Systems Engineering Research Center, Korea Research Institute of Bioscience and Biotechnology, Daejeon, 34141, Korea

E. J. Park

Seaweed Research Center, National Fisheries Research and Development Institute, Mokpo, 58746, Korea

\section{Introduction}

Temperature is a major environmental factor that limit the growth and yield of plant. Under high temperatures or heat stress, plants alter gene expression patterns to adapt to a given environment. Heat shock protein (HSP) genes are key components turned on under heat stress condition and contributing to cellular homeostasis (Scharf et al. 2001; Wang et al. 2004; Schroda and Vallon, 2009; Basha et al. 2012). They are responsible for protein folding, assembly, translocation and degradation in a broad array of normal cellular processes; they also function in the stabilization of proteins and membranes, and can assist in protein refolding under stress conditions. All organism response to high temperature and turning on the HSPs which are a number of conserved protein families such as the HSP100s, HSP90s, HSP70s, HSP60s and sHSP (Wang et al. 2004).

The small heat shock protein (sHSP) is a family of heat shock protein that range in size from approximately 12 42 $\mathrm{kDa}$. Most sHSPs are in the range of $15 \sim 22 \mathrm{kDa}$. This protein is characterized by having a conserved C-terminal domain of approximately 90 amino acids referred to as the a-crystallin domain (ACD) (Basha et al. 2012; Scharf et al. 2001; Waters, 2013). Except ACD domain, sHSP are variable in both length and sequence. The sHSPs that function in specific cellular organelles or compartments have $\mathrm{N}$-terminal transit or signal sequences needed to get the sHSP to the proper cellular compartment (Waters, 2013).

The high resolution crystal structures studies show that sHSP are composed of a $\beta$-sandwich of two antiparallel sheets and form a hollow ball (van Montfort et al. 2002; Waters, 2013; Zhang et al. 2015). The oligomers are of different sizes. The plant Hsp16.9 from wheat (Triticum aestivum) (van Montfort et al. 2001; 2002) forms a dodecamer, and the oligomer of Hsp16.5 from an archaea, Methano 
caldococcus (Methanococcus) jannaschii (Kim et al. 1998) has 24 subunits. (van Montfort et al. 2001). A model for the structure of Hsp21 from Arabidopsis indicates that this plant sHSP is also a dodecamer with two hexameric disks (Lambert et al. 2011). Stengel et al. (2010) showed that the dodecamer form of Hsp18.1CI from Pisum sativum is in equilibrium with Hsp18.1 dimers, monomer, and higher order oligomers.

The sHSPs are ubiquitously present in all organisms. The number of genes encoding sHSPs in different organisms varies greatly. Investigations of sHSPs from mammals, yeast, plants and bacteria have unveiled their role in thermotolerance such that the overexpression of eukaryotic recombinant sHSPs could increase the thermotolerance of $E$. coli cells. The plant sHSPs are particularly diverse and are a crucial component of the plant heat shock response.

The sHSP are known to act as ATP-independent molecular chaperones work with other chaperones to prevent irreversible aggregations and to re-solubilize proteins that have already aggregated (Waters, 2013; Zhang et al. 2015). They do not require ATP to bind substrate proteins. The sHSP bind to the non-native substrate proteins and release them for refolding with the help of other ATP-dependent chaperones (Waters, 2013). In the absence of such stresses, however, sHSPs can also be produced specifically in reproductive organs at certain developmental stages, including seed maturation and germination, pollen development, and fruit maturation (Neta-sharir et al. 2005; Volkov et al. 2005).

Pyropia (Bangiales, Rhodophyta) are one of the most commercially valuable marine red algae; it is used as a source of food, fertilizer, medicine, and chemicals (Sahoo et al. 2002). Among them, several species have been cultured in aquaculture industry in East Asia including Korea (Hwang et al. 2005). Pyropia grow naturally in the intertidal zone, where they are frequently exposed to a variety of potentially stressful environmental including variations in humidity, changes in temperature, osmotic shock and different light intensities (Hwang et al. 1997; Blouin et al. 2011). Therefore, Pyopia have developed a variety of strategies and mechanisms to respond to and survive through those environmental stresses (Choi et al. 2013; Im et al. 2015). Pyropia exhibits a exhibits a heteromorphic life cycle with an alternation of generation between foliose thallus gametophyte and filamentous sporophyte; this is referred to as conchocelis phase (Sahoo et al. 2002, Blouin et al. 2011). The thallus that we see and use as a food source is the haploid generation, and it can reproduce asexually by forming spores that grow to replicate the original thallus. Gametophyte thalli are grown in cold water during the winter season and changed into conchocelis phase for summer. Temperature is one of the major factors that affect growth rate and life cycle of the Pyropia (Hwang et al. 1997). The increase of seawater temperature due to global warming affect the growth of Pyropia. However, information currently known regarding genes affecting the regulatory and biochemical pathways of high temperature responses in Pyropia is limited. Identifying genes and studying their expression patterns and physiological function in response to high temperature will provide a molecular basis for life cycle as well as heat tolerance of the Pyropia.

We identified four small heat shock genes ( $s H S P$ ) based on transcriptome generated from $P$. tenera thalli under normal and high-temperature condition. In this study, we report that expression pattern of PtsHPS19.3 and its physiological function in heat and abiotic stress tolerance in heterologous expressed Chlamydomonas cells.

\section{Material and Methods}

Plant materials and growth condition

Pyropia tenera (LB) was provided from Seaweed research center at Mokpo, Korea. Leafy gametophytes of $P$. tenera were cultured in modified Grund medium (McLachlan, 1973) at $12^{\circ} \mathrm{C}$ under irradiation with $80 \mu \mathrm{mol}$ photon $\mathrm{m}^{-2} \mathrm{~s}^{-1}$, provided by cool-white fluorescent lamps on a 10:14 (light: dark) photoperiod in a growth room. Growth bottles containing $P$. tenera were transferred to a $20^{\circ} \mathrm{C}$ growth chamber with the same light intensity and photoperiod for the heat treatment. Desiccation and freezing stress were applied as described by Im et al. (2015).

Chlamydomonas reinhardtii strain mut-11 $(\mathrm{mt}+)$ was grown in Tris-acetate-phosphate (TAP) medium at $25^{\circ} \mathrm{C}$ with shaking at $100 \mathrm{rpm}$ under continuous cool fluorescent light $(50 \mu \mathrm{mol}$ photon $\left.\mathrm{m}^{-2} \mathrm{~s}^{-1}\right)$.

\section{PtsHSP 19.3 cDNA isolation and sequence analysis}

Transcriptome sequence reads generated from $P$. tenera gametophyte thalli were analyzed for the sHSP gene family (Choi et al. 2013). The contigs encoding putative sHSPs were analyzed to determine if they covered the full open reading frame (ORF). Finally, four contigs encoding putative full $\mathrm{s} H S P$ ORFs were selected for further analysis. Gene-specific primer sets were designed for each contig to clone the $\mathrm{s} H S P$ cDNA, and the PCR products were cloned into the pGEM T-Easy vector (Promega, Madison, WI, USA). Plasmid DNA 
was purified using a Qiaquick Plasmid Extraction Kit (Qiagen, Hilden, Germany) and sequenced.

Sequence editing and amino acid sequence prediction from the selected contigs were conducted using the Sequencher program (Gene Code Corp., Ann Arbor, MI, USA). The putative molecular weights and $\mathrm{pI}$ values of the deduced polypeptides were predicted using the Compute $\mathrm{pI} / \mathrm{Mw}$ program (http://web.expasy.org/compute pi/). The deduced amino acid sequences were aligned using the ClustalX program (http://www.ebi.ac.uk/clustalw). Phylogenic trees for sHSPs from green plants and PtsHSP19.3 were built with the neighbor-joining method using the Geneious program (Geneious, Auckland, New Zealand). Conserved motifs or domains were predicted using the Prosite program (http:// expasy.org/prosite).

Gene expression analysis

Gene-specific qRT-PCR was conducted to assay PtsHSP19.3 gene expression patterns. Total RNA was prepared from gametophyte thalli using a Plant RNeasy kit (Qiagen, Hilden, Germany). First-strand cDNA was constructed from about $2 \mu \mathrm{g}$ of total RNA via reverse-transcription in $20 \mu \mathrm{L}$ reaction volumes, using the oligo $(\mathrm{dT})_{17}$ primer, amfiRivertII cDNA Synthesis $2 \times$ Reaction Buffer with the oligo $(\mathrm{dT})_{17}$ primer, and the cDNA Synthesis Enzyme Mix (GenDepot, Barker, TX, USA), according to the manufacturer's instructions. The reactions were conducted for $5 \mathrm{~min}$ at $25^{\circ} \mathrm{C}$ and $60 \mathrm{~min}$ at $50^{\circ} \mathrm{C}$, followed by $15 \mathrm{~min}$ of heating at $70^{\circ} \mathrm{C}$. The first-strand cDNA reaction was diluted by a factor of 10 , and $2 \mu \mathrm{L}$ of the dilution was applied for PCR-amplification in a reaction containing $5 \mu \mathrm{L}$ of $10 \times$ PCR buffer $(200 \mathrm{mM}$ Tris-HCl, $\mathrm{pH}$ 8.4, and $500 \mathrm{mM} \mathrm{KCl}$ ), $1 \mu \mathrm{L} 10 \mathrm{mM}$ dNTPs, $1 \mu \mathrm{L}$ of each gene-specific primer $(10 \mu \mathrm{M})$, and 2.5 units of ExTaq DNA polymerase (Takara, Shiga, Japan). The PCR reactions were carried out for 35 cycles of $30 \mathrm{sec}$ at $95^{\circ} \mathrm{C}, 30 \mathrm{sec}$ at $58 \sim$ $64^{\circ} \mathrm{C}$, depending on the primers, and $60 \mathrm{sec}$ at $72^{\circ} \mathrm{C}$, followed by $5 \mathrm{~min}$ of termination at $72^{\circ} \mathrm{C}$. The gene-specific primers were as follows: $5^{\prime}$-CTCTCTCCCATCACACGACTC- $3^{\prime}$ and 5'-GAACCCAGGTGTCTCGAACC-3' for PtsHSP19.3. The PCR products were separated on $1 \%$ agarose gels and stained with ethidium bromide for imaging.

qRT-PCR was carried out on a Rotor-Gene RG-3000 cycler (Corbett, Sydney, Australia) using the QuantiTect SYBR Green PCR kit (Qiagen), according to the manufacturer's instructions. The qRT-PCR program consisted of a predenaturation step at $95^{\circ} \mathrm{C}$ for $10 \mathrm{~min}$ and 40 cycles of amplification at $95^{\circ} \mathrm{C}$ for $15 \mathrm{sec}, 60^{\circ} \mathrm{C}$ for $30 \mathrm{sec}$, and $72^{\circ} \mathrm{C}$ for 30 sec. All samples were run in duplicate and n-fold differential expression was calculated using the comparative Ct method, $2^{-\Delta \Delta \mathrm{Ct}}$. The actin (5'-GGTGCAGAAGGAGG TGGAGA- $3^{\prime}$ and $5^{\prime}$-GCAACGCAACCCATAGAGAA- $3^{\prime}$ ) gene was used as an internal control. All samples were run in duplicate, and n-fold differential expression was calculated using the comparative $\mathrm{Ct}$ method. N-fold differential expression was calculated by $2^{-\Delta \Delta \mathrm{Ct}}$.

Cellular localization of PtsHSP19.3

The PtsHSP19.3 coding region was amplified via PCR with ExTaq DNA polymerase (Takara, Shiga, Japan) using forward (5'-TCTAGAATGGACCTTTTCGCACTTGA-3') and reverse (5'-GGATCCCTACTCAGTCACCTGCGGTC-3') primers containing an $X b a \mathrm{I}$ site upstream and a BamHI site downstream and was introduced into the $X b a \mathrm{I} / \mathrm{BamHI}$ site of the 326 GFP-3G vector. Recombinant DNA was introduced into tobacco (Nicotina benthamiana) protoplasts. The tobacco protoplasts were examined under a fluorescence microscope (Leica, Wetzlar, Germany) to evaluate GFP expression.

Stress tolerance assay for the PtsHSP19.3 gene in Chlamydomonas

The PtsHSP19.3 gene ORF was amplified via PCR and subcloned under the PtsHSP19.3 pCr112 vector using the NdeI and EcoRV sites. The pCr112-PtsHSP19.3 plasmid was introduced into Chlamydomonas strain mut-11 via the glass bead method for transformation. Transformants were selected on TAP agar medium containing $20 \mu \mathrm{g} \mathrm{mL}^{-1}$ hygromycin after $14 \sim 16$ days of growth. Chlamydomonas genomic DNA was purified from 100-ml liquid cultures and used for PCR with the PtsHSP19.3-specific primer set to confirm insertion of the PtsHSP19.3 gene into the Chlamydomonas genome. Total RNA was isolated from control and transformed cells and applied to RT-PCR to assess PtsHSP19.3 gene expression in transgenic Chlamydomonas cells. The $C r$ actin (5'-TGTGCATACGTGGATAGCTTG-3' and 5'-ATGACCCGCTCCTCATATCTT-3') gene was used as an internal control.

Chlamydomonas cells were grown in TAP medium containing hygromycin at $25^{\circ} \mathrm{C}$ under a $14 \mathrm{~h} \mathrm{~d}^{-1}$ photoperiod. The cells were first grown at a concentration of $2 \sim 4 \times 10^{6}$ cells $\mathrm{mL}^{-1}$ and concentrated to $10^{7}$ cells $\mathrm{mL}^{-1}$ before dilution to $10^{0} \sim 10^{-2}$ in fresh medium. The diluted cells $(8 \mu \mathrm{L})$ were inoculated onto agar plates. To evaluate high temperature tolerance, the cells were exposed to $38^{\circ} \mathrm{C}$ for $1 \sim 3$ days, and transferred to a $25^{\circ} \mathrm{C}$ growth chamber. C. reinhardtii strain mut-11 and the transformed cells with the pCr112 
vector, Hyg5414, were used as controls. To check osmotic, oxidative or salt tolerance, cells were inoculated onto agar medium with $200 \mathrm{mM}$ Mannitol, $0.03 \% \mathrm{H}_{2} \mathrm{O}_{2}$ or $50 \mathrm{mM}$ $\mathrm{NaCl}$.

\section{Results and Discussion}

Identification and characterization of the $P$. tenera sHSP 19.3 gene

The transcriptome sequences from $P$. tenera gametophyte thalli have been generated under normal growth and heat stress conditions (Choi et al. 2013), and attempts were made to identify the differentially expressed genes (DEG) under heat stress conditions. Among the identified DEGs, the most popular and well-known genes were small heat shock proteins (sHSPs), which constitute a small and highly conserved family in eukaryotes and prokaryotes and are highly involved in the heat shock response (Choi et al. 2013; Waters, 2013). The transcriptome sequences were screened for sHSP members, and 19 contigs encoding putative alpha crystalline domain (a-crystalline, ACD) were obtained. The open reading frame (ORF) findings and results from homolog searches showed that many of the contig sequences were truncated at the $5^{\prime}$ - or $3^{\prime}$-regions. Finally, four unique sequences covering full ORF were selected (Table 1). These results indicate that there are more than four $s H S P$ genes in the $P$. tenera genome. The characteristics of the four PtsHSP polypeptides are summarized in Table 1. All PtsHSP polypeptides had molecular weights of $16.4 \sim 20.2 \mathrm{kDa}$ and isoelectric points of $4.7 \sim 6.42$. Amino acid sequence alignment of the PtsHSP polypeptides showed sequence variations, including deletions and substitutions (Fig. 1A). An ACD domain was detected in all identified PtsHSPs. Amino acid homology searching by Blast program result show that four PtsHSPs have significant homology with previously deposited PhsHSP22 from $P$. haitanensis, red algae (Table 1). PtsHSP19.3 have the highest sequence homology with
PhsHSP22 from $P$. haitanensis (Table 1). Therefore we chose the PtsHSP19.3 for further analysis of its expression and physiological function.

Except PhsHSP22, there are no known genes showing significant amino acid sequence homology with PtsHSPs in public database. These results indicate that red algae sHSPs are much different from the known sHSPs from green plants including single cell algae and higher land plants (Fig 1B). Or PtsHSP19.3 may have no chaperon activity although ACD domain was found.

\section{Expression of PtsHSP 19.3 genes}

A characteristic of many $\mathrm{s} H S P$ genes is their strong and universal induction in response to heat stress. Several sHSPs are also induced by other biotic and abiotic stressors, such as drought and cold (Wehmeyer and Vierling, 2000; Waters, 2013). Total RNA was isolated after $P$. tenera was subjected to heat, low temperature, desiccation, and high salt condition, and quantitative reverse transcription-polymerase chain reaction (qRT-PCR) analyses were conducted to evaluate the expression of each PtsHSP 19.3 gene. Figure 2 shows the expression of each PtsHSP 19.3 gene under heat, desiccation and freezing stress conditions. PtsHSP19.3 responded to heat stress (Fig. 2a). But PtsHSP 19.3 response slightly or not to desiccation, freezing or high salt condition. Expression of the PtsHSP 19.3 was increased until 8 hours after heat treatment and then decreased (Fig. 2b). The most common expression pattern of $s H S P$ gene in model plant Arabidopsis thaliana is no expression in unstressed tissue and high level of expression during heat stress (Waters, 2013). There is no single pattern of gene expression for $s H S P$ genes. Most of the Arabidopsis sHSP genes response to various abiotic stress including osmotic, oxidative, salt and wounding stress as well as heat (Malik et al. 1999; Lee et al. 2000; Sun et al. 2001; Waters et al. 2008). Example, class I sHSPs of Arabidopsis are highly expressed during heat stress, but they are also expressed in response to a wide range of other stressors including osmotic stress, oxidative stress and UV. But expression level of the each Arabidopsis sHSP gene

Table 1 Summary of small heat shock proteins (PtsHSPs) isolated from P. tenera

\begin{tabular}{ccccccc}
\hline Gene & contig name & $\begin{array}{c}\text { No. amino acid } \\
\text { residues }\end{array}$ & $\begin{array}{c}\text { MW } \\
(\mathrm{kDa})\end{array}$ & pI & e-value & Description \\
\hline PtsHSP19.3 & Ptcontig714 & 173 & 19.3 & 5.44 & $6 \mathrm{e}-96$ & HSP22 of Pyropia haitanensis \\
PtsHSP19.6 & Ptcontig6407 & 179 & 19.6 & 6.05 & $3 \mathrm{e}-72$ & HSP22 of Pyropia haitanensis \\
PtsHSP20.2 & Ptcontig6022 & 200 & 20.2 & 6.42 & $7 \mathrm{e}-21$ & HSP22 of Pyropia haitanensis \\
PtsHSP16.4 & Ptcontig3221 & 154 & 16.4 & 4.70 & $1 \mathrm{e}-19$ & HSP of Galdieria sulphuraria \\
\hline
\end{tabular}




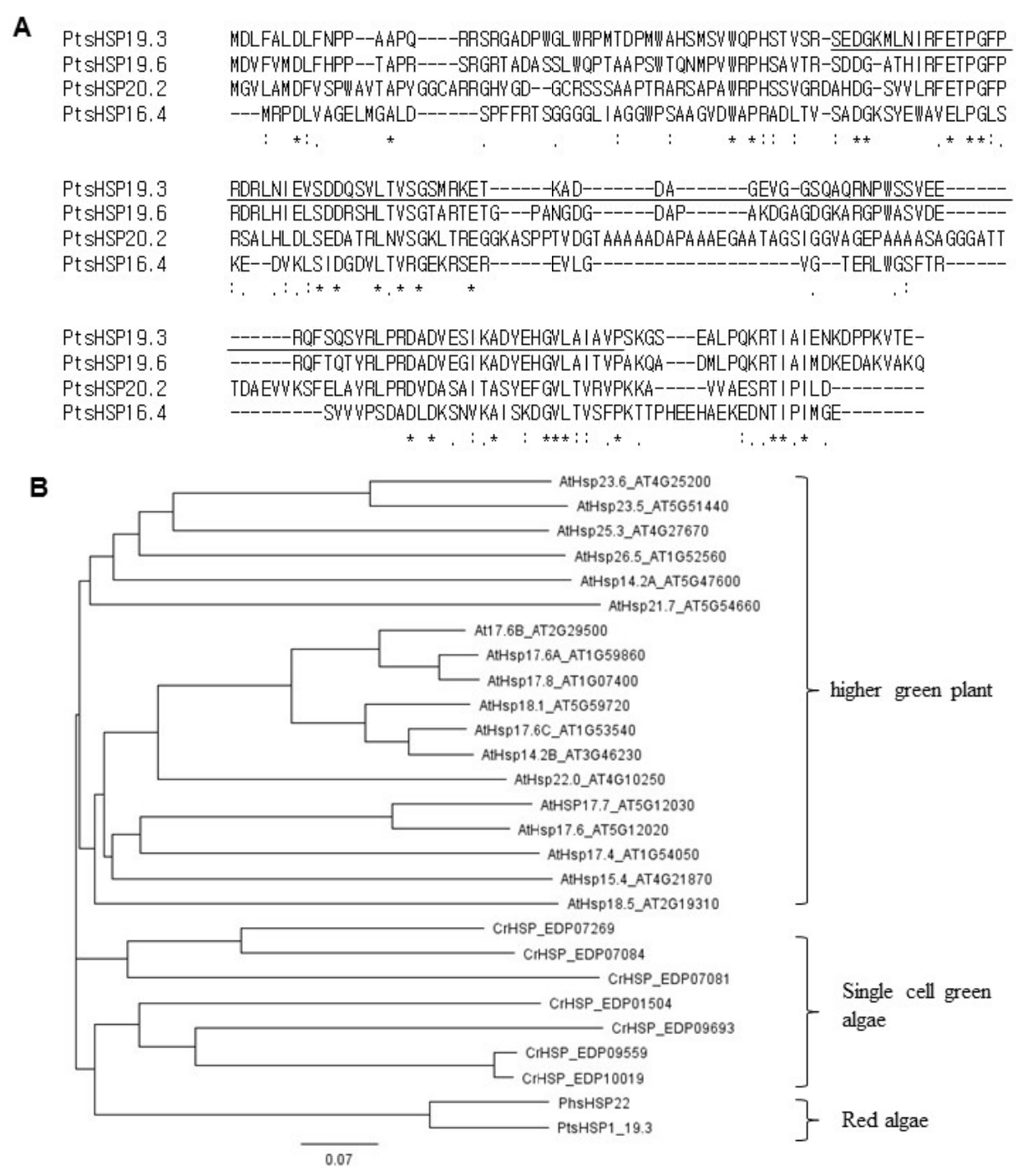

Fig. 1 Amino acid sequence alignment and phylogenic tree of PtsHSPs isolated from $P$. tenera. A, ACD domain identified in PtsHSP19.3 was underlined. Asterisk $(*)$ and : indicate identical and similar amino acid residues, respectively. The alignment was conducted using the CLUSTAL W. B, Phylogenic tree of sHSPs. Two sHSP, PtsHSP19.3 and PhsHSP from Pyropia (red algae), sHSPs from Chlamydomonas (single cell green algae) and Arabidopsis (higher green plant) were selected for phylogenic analysis

differs depending on the type of abiotic stress. In addition to being heat induced, some $s H S P s$ are expressed during embryogenesis and seed maturation (Wehmeyer and Vierling, 2000; Sun et al. 2002; Kotak et al. 2007).

\section{Localization of PtsHSP 19.3}

To determine the cellular location of the PtsHSP 19.3 protein, the PtsHSP 19.3 ORF sequence was fused upstream of a reporter gene encoding GFP in the plant expression vector p326-35S-GFP (GFP). The recombinant vector, p326-35SPtsHSP19.3-GFP (PtsHSP19.3-GFP), was then subjected to a transient assay using tobacco protoplast. The p326-GFP vector showed GFP signals in both the cytoplasm and cell organelles including nucleus (Fig. 3). The p326-PtsHSP19.3-
GFP showed GFP signals in the several locations as spots or granules (Fig. 3). Prediction of the signal peptides using the PrediSi program (www.pridisi.de) demonstrated that PtsHSP19.3 do not harbor a putative signal peptide in the $\mathrm{N}$-terminal region for subcellular organelle. These results suggest that PtsHSP19.3 may be in cytosol and not located in cell organelles such as nucleus, chloroplast or mitochondria.

Plant have more sHSPs than other eukaryotes (Waters et al. 2008; Yan et al. 2017). Analysis of three angiosperm genome sequence including Arabidopsis thaliana, Populus trichocarpa and Oryza sativa show that there are 11 sHSP subfamilies (Waters et al. 2008). Among them, six subfamilies are localized in cytoplasm/nuclear and five sHSP subfamilies localized cellular organelles including endoplasmic reticulum (ER), chloroplast, mitochondria, and peroxisome. The sHSPs 
A

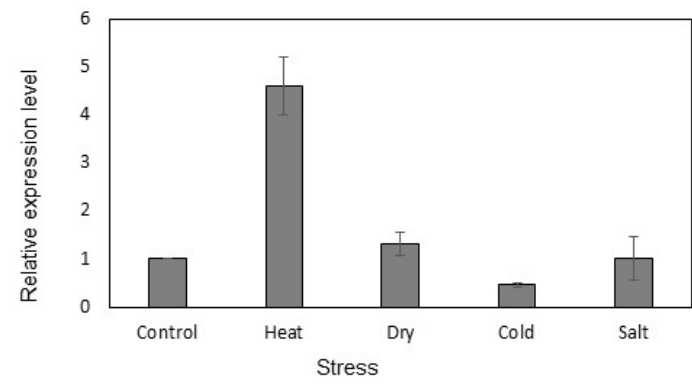

B

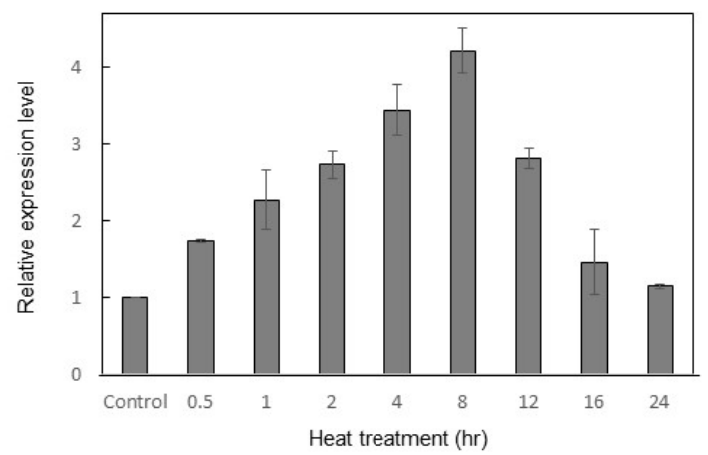

Fig. 2 Expression pattern of the PtsHSP19.3 gene in P. tenera. A, Total RNAs were purified from the gametophyte thalli of $P$. tenera under heat, desiccation, cold or salt stress conditions and amplified by qRT-PCR. The PtAct gene was used as internal control. Bars are means \pm standard deviation (SD). B, To check the expression pattern of the PtsHSP19.3 gene during heat stress, total RNA was purified from the gametophyte thalli of $P$. tenera at indicated point after heat treatment

that function in specific cellular organelles or compartments have N-terminal transit, leader, or signal sequences needed to get the sHSP to the proper cellular compartment.

Arabidopsis study reported that some cytosolic sHSPs localize as multichaperone complex in cytosol (Siddique et al. 2008). The sHSPs of the cytoplasmic/nuclear subfamilies CI, CII and CIII are shown to be recruited to heat shock complex under heat stress condition (Siddique et al. 2008). Recently Zhang et al (2015) reported that small heat shock protein CeHSP17 from Caenorhabditis elegans exists as a 24 subunit spherical oligomer and forms large sheet like super molecular assemblies at the high temperature. These results suggest that PtsHSP 19.3 may form large super molecular assemblies in cytosol or located in heat stress granule.

Expression of the PtsHSP 19.3 gene increase heat and abiotic tolerance in Chlamydomonas

The complete PtsHSP19.3 coding sequence was introduced into $C$. reinhardtii strain mut11 to assess the physiological functions of the PtsHSP19.3 gene under heat, cold, high

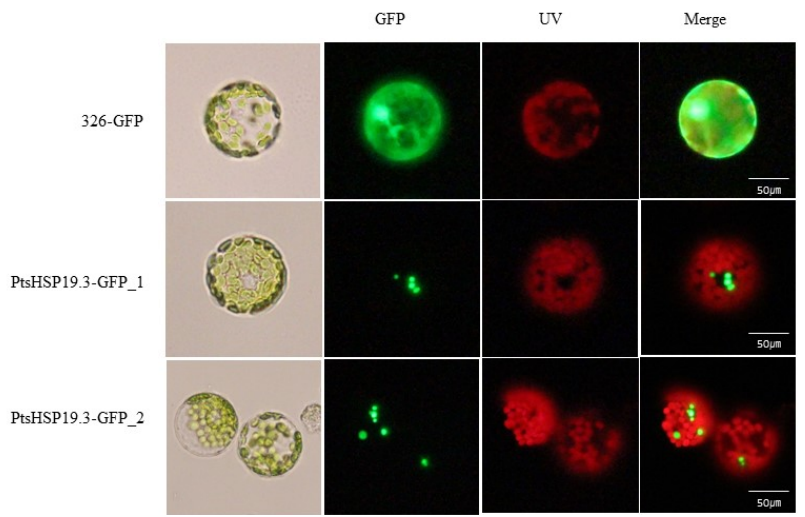

Fig. 3 Subcellular localizations of PtsHSP19.3

A reporter gene encoding green fluorescent protein (GFP) was fused to PtHSP19.3 under the control of the CaMV 35S promoter in the 326-GFP vector. The constructs were introduced into the protoplasts of Nicotiana benthamiana. The 326-GFP empty vector served as the control. After overnight incubation, PtsHSP19.3-GFP localization in individual cells was observed by GFP fluorescence. Contrast interference images of the structure of the whole protoplast were presented at left. GFP, cell images using a green filter for PtsHSP19.3-GFP location with GFP fluorescence. Different protoplast (PtsHSP19.3-GFP_1 and PtsHSP19.3-GFP_2) expressing GFP are presented. UV, cell images using a UV filter for chloroplast location. Merge, merged cell image from GFP and UV filter

salt, and osmotic stress conditions. Three PtsHSP19.3transformed Chlamydomonas lines were selected on media containing hygromycin, and introduction and expression of the PtsHSP19.3 gene were confirmed via PCR (Fig. 4A) and RT-PCR (Fig. 4B). In order to assess the thermotolerance conferred by PtsHSP19.3, transformed Chlamydomonas lines were subjected or not (control) to a $38^{\circ} \mathrm{C}$ for 2 days and then they were transferred to a $25^{\circ} \mathrm{C}$ incubator and cultured for 10 days. Chlamydomonas strain Mut11 and Hyg5414 transformed by empty vector were used as control. All Chlamydomonas cells had grown to similar levels as those observed on control culture plates (Fig. 4C). However, the transformed Chlamydomonas lines showed a much higher growth rate than that of the Hyg5414, Mut 11 line when heat stress was applied. These results demonstrate that PtsHSP19.3 contributes to heat stress tolerance in Chlamydomonas. However, when cold temperature was applied to Chlamydomonas lines, transformed Chlamydomonas grown similar level with control cells (data not shown). These results indicate that PtsHSP19.3 does not involved in cold tolerance. Transformed Chlamydomonas also does not show tolerance to osmotic stress induced by mannitol (data not shown). These results are correlated with expression pattern of the PtsHSP19.3 (Fig 2). qRT-PCR result show that PtsHSP19.3 does not response to low temperature and desiccation stress. When the transformed Chlamydomonas were subjected into 


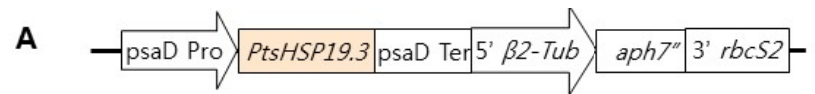
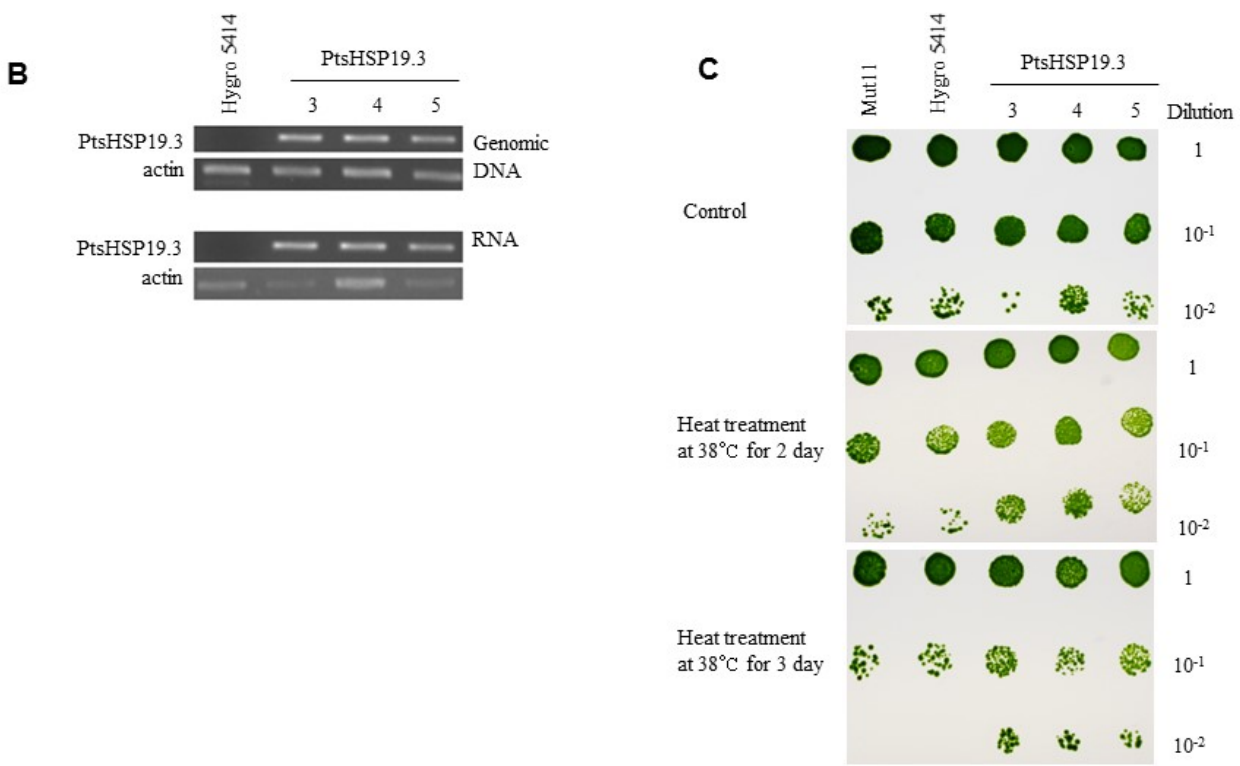

Fig. 4 Effects of PtsHSP19.3 on growth of Chlamydomonas under heat stress conditions

A. Vector map for the expression of PtsHSP19.3 in Chlamydomonas. B, To evaluate the introduction and expression of the PtsHSP19.3 gene, genomic DNAs and RNA were purified from transgenic Chlamydomonas cells and amplified with a PtsHSP19.3 specific primer. $\mathrm{C}$, Survival and growth assay of the transgenic Chlamydomonas cells harboring PtsHSP19.3 under heat stress. Chlamydomonas cells were diluted to $10^{-1} \sim 10^{-3}$ in fresh medium and $10 \mu \mathrm{l}$ of diluted cells were inoculated onto agar plates. The Chlamydomonas cells were subjected or not (control) to a $38^{\circ} \mathrm{C}$ for 2 or 3 days and transferred to a $25^{\circ} \mathrm{C}$ incubator and cultured for 10 days. The Chlamydomonas strain Mut11 and Hyg5414 transformed by empty vector were used as control

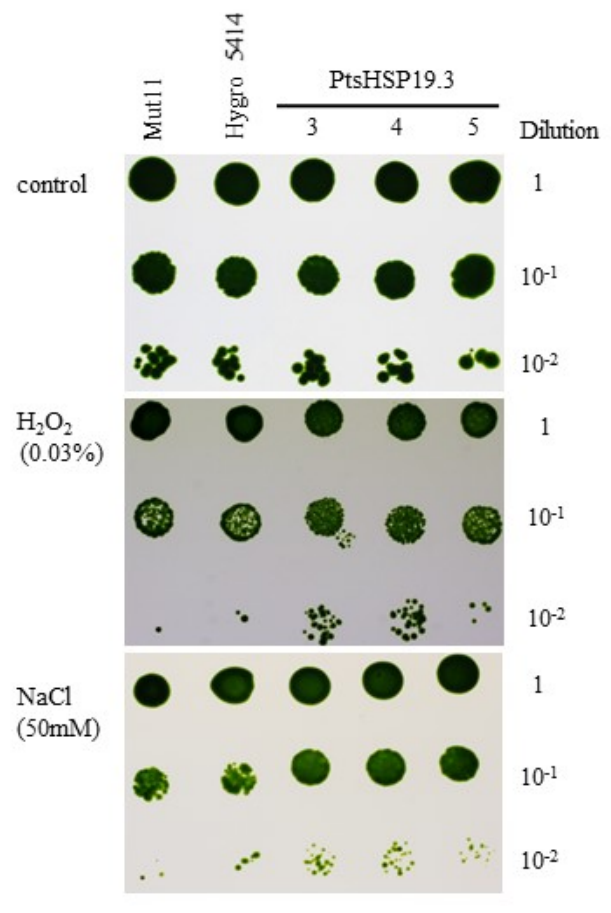

Fig. 5 Effects of PtsHSP19.3 on growth of Chlamydomonas under abiotic stress condition. Chlamydomonas cells were diluted and inoculated onto agar plates containing $0.03 \% \mathrm{H}_{2} \mathrm{O}_{2}$ or 50 $\mathrm{mM}$ salt as described in Fig 4 medium containing $50 \mathrm{mM}$ salt or $0.03 \% \mathrm{H}_{2} \mathrm{O}_{2}$, transformed cells showed a much higher growth rate than that of the control cells (Fig. 5). These results demonstrate that PtsHSP19.3 contributes to oxidation stress and salt tolerance in Chlamydomonas. Previous studies in higher plants show that sHSPs are involve in salt and oxidation stress tolerance as well as high temperature (Malik et al. 1999; Lee et al. 2000; Sun et al. 2001; $\mathrm{Mu}$ at al. 2013; Ruibal et al. 2013). When a sHSP from lily, LimHSP16.46, was overexpressed in Arabidopsis, transgenic plant enhanced in viability under high temperature, high salt and oxidative stresss (Mu et al. 2013). Overexpression of a sHSP, JrsHSP17.3 from walnut tree (Juglans regia) confer the tolerance to heat, freezing and salt stress in transgenic yeast (Zhai et al. 2016). These result demonstrate that PtsHSP19.3 from red algae plays a role in abiotic stress tolerance including high temperature as $s H S P s$ in higher plants, although, PtsHSP19.3 have low amino acid sequence homology to sHSP of higher plants.

\section{Conclusion}

Pyropia tenera is one of the most commercially valuable 
marine red algae. We identified four putative small heat shock protein genes ( $S H S P)$ based on transcriptome generated from $P$. tenera. Although ACD domain was found, they have no significant amino acid sequence homology with known gene in public database. The PtsHSP19.3 gene was highly upregulated by high temperature, and confer heat tolerance in Chlamydomonas. The PtsHSP 19.3 polypeptide may form large super molecular assemblies or located in stress granule in cytosol. Global warming affect increasing seawater temperature. Water temperature is one of the major factor affecting on the growth and life cycle in Pyropia tenera. This study provide information for sHSPs and its function in heat tolerance of Pyropia.

\section{Acknowledgments}

This work was supported by Korean Institute of Planning and Evaluation for Technology, Agriculture, Forestry and Fisheries (IPET) through Golden Seed Project (Project number, 213008-05-1-SB830), funded by Ministry of Agriculture, Food, and Rural Affairs (MAFRA); the Ministry of Oceans and Fisheries (MOF); the Rural Development Administration (RDA); and the Korea Forest Service (KFS).

\section{References}

Basha E, O’Neill H, Vierling E (2012) Small heat shock proteins and alpha-crystallins: dynamic proteins with flexible functions. Trends Biochem Sci 37:106-117

Blouin NA, Brodie JA, Grossman AC, Xu P, Brawley SH (2011) Porphyra: a marine crop shaped by stress. Trends Plant Sci 16:29-37

Choi S, Hwang MS, Im SO, Kim NJ, Jeong WJ, Park EJ, Gong YG, Choi DW (2013) Transcriptome sequencing and comparative analysis of the gametophyte of Pyropia tenera under normal and high-temperature condition. J Appl Phycol 25:1237-1246

Hwang MS, Chung IK, Oh YS (1997) Temperature responses of Porphyra tenera Kjellman and P. yezoensis Ueda (Bangiales, Rhodophyta) from Korea. Algae 12:207-213

Hwang MS, Kim SM, Ha DS, Baek JM, Kim HS, Choi HG (2005) DNA sequences and identification of Porphyra cultivated by natural seeding on the southwest coast of Korea. Algae 20:183-196

Im S, Choi S, Hwang MS, Park EJ, Jeong WJ, Choi DW (2015) De novo assembly of transcriptome from the gametophyte of the marine red algae Pyropia seriata and identification of abiotic stress response genes. J Appl Phycol 27:1343-1353

Kim KK, Kim R, Kim S-H (1998) Crystal structure of a small heat shock protein. Nature 394:595-599

Kotak S, Vierling E, Baumlein H, von Koskull-Doring P (2007) A novel transcriptional cascade regulating expression of heat stress proteins during seed development of Arabidopsis. Plant Cell 19:182-195

Lambert W, Koeck PJ, Ahrman E, Purhonen P, Cheng K, Elmlund D, Hebert H, Emanuelsson C (2011) Subunit arrangement in the dodecameric chloroplast small heat shock protein Hsp21. Protein Science 20:291-301

Lee BH, Won SH, Lee HS, Miyao M, Chung WI, Kim IJ, Jo JK (2000) Expression of the chloroplast-localized small heat shock protein by oxidative stress in rice. Gene 245:283-290

Malik MK, Slovin JP, Hwang CH, Zimmerman JL (1999) Modified expression of a carrot small heat shock protein gene, $h s p 17.7$, results in increased or decreased thermotolerance double danger. Plant J 20:89-99

McLachlan J (1973) Growth media-marine. In: Stein JR (ed) Handbook of phycological methods, Cambridge University Press, New York

Mu C, Zhang S, Yu G, Chen N, Li X, Liu H (2013) overexpression of small heat shock protein LimHSP16.45 in Arabidopsis enhances tolerance to abiotic stresses. Plos One 8:12

Neta-Sharir I, Isaacson T, Lurie S, Weiss D (2005) Dual role for tomato heat shock protein 21: protecting photosystem II from oxidative stress and promoting color changes during fruit maturation. Plant Cell 17:1829-1838

Ruibal C, Castro A, Carballo V, Szabados L, Vidal S (2013) Recovery from heat, salt and osmotic stress in Physcomitrella patens requires a functional small heat shock protein PpHsp16.4. BMC Plant Biol 13:174

Sahoo D, Tang X, Yarish C (2002) Porphyra-the economic seaweed as a new experimental system. Curr Sci India 83: 1313-1316

Scharf KD, Siddique M, Vierling E (2001) The expanding family of Arabidopsis thaliana small heat stress proteins and a new family of proteins containing alpha-crystallin domains Acd proteins. Cell Stress \& Chaperones 6:225-237

Schroda M, Vallon O (2009) Chaperones and proteases. In: Stern DB (ed) Chlamydomonas source book $2^{\text {nd }}$ edn, volume 2, Elsevier, San Diego

Siddique M, Gernhard S, von Koskull-Doring P, Vierling E, Scharf KD (2008) The plant sHSP superfamily: five new members in Arabidopsis thaliana with unexpected properties. Cell Stress and Chaperones 13:183-17

Stengel F, Baldwin AJ, Painter AJ, Jaya N, Basha E, Kay LE, Vierling E, Robinson CV, Benesch JLP (2010) Quaternary dynamics and plasticity underlie small heat shock protein chaperone function. Proc Nat Acad Sci USA 107:2007-2012

Sun W, Van Montagu, Verbruggen N (2002) small heat shock proteins and stress tolerance in plants. Biochemica et Biophysica Acta 1577:1-9

Sun W, Bernard C, van de Cotte B, van Montagu M, Verbruggen N (2001) At-HSP17.6A, encoding a small heat-shock protein in Arabidopsis, can enhance osmotolerance upon overexpression. Plant J 27:407-415

Van Montfort R, Slingsby C, Vierling E. (2002) Structure and function of the small heat shock protein/alpha-crystallin 
family of molecular chaperones. Advances in Protein Chem 59:105-156

Van Montfort RL, Basha E, Friedrich KL, Slingsby C, Vierling E (2001) Crystal structure and assembly of a eukaryotic small heat shock protein. Nature Structural Biology 8:1025-1030

Volkov RA, Panchuk II, Schoffl F (2005) Small heat shock proteins are differentially regulated during pollen development and following heat stress in tobacco. Plant Mol Biol 57:487-50

Wang W, Vinocur B, Shoseyov O, Altman A (2004) Role of plant heat-shock proteins and molecular chaperons in the abiotic stress response. Trends Plant Sci 9:244-252

Waters ER (2013) The evolution, function, structure, and expression of the plant sHSPs. J Exp Bot 64:31.403

Waters ER, Aevermann BD, Sanders-Reed Z (2008) Comparative analysis of the small heat shock proteins in three angiosperm genomes identifies new subfamilies and reveals diverse evolutionary patterns. Cell Stress \& Chaperones 13:127-142
Wehmeyer N, Vierling E (2000) The expression of small heat shock proteins in seeds responds to discrete developmental signals and suggests a general protective role in desiccation tolerance. Plant Physiol 122:1099-1108

Zhai M, Sun Y, Jia C, Peng S, Liu Z, Yang G(2016) Over-expression of JrsHSP17.3 gene from Juglans regia confer the tolerance to abnormal temperature and $\mathrm{NaCl}$ stresses. J Plant Biol 59:549-558

Zhang K, Ezemaduka AN, Wang Z, Hu H, Shi X, Liu C, Lu X, Fu X, Chang Z, Yin CC (2015) A Novel Mechanism for Small Heat Shock Proteins to Function as Molecular Chaperones. Scientific reports. 5:8811

Yan H, Zhang A, Chen J, He X, Xu B, Xie G, Miao Z, Zhang X, Huang L (2017) Geneome-wide analysis of the PvHsp20 family in switchgrass: motif, genomic organization, and identification of stress or developmental-related Hsp20s. Front Plant Sci 8:1024 